The Astrophysical Journal, 274:231-236, 1983 November 1

(C) 1983. The American Astronomical Society. All rights reserved. Printed in U.S.A.

\title{
GAMMA-RAYS FROM ATOMIC AND MOLECULAR GAS IN THE FIRST GALACTIC QUADRANT
}

F. Lebrun, ${ }^{1,2}$ K. Bennett, ${ }^{3}$ G. F. Bignami, ${ }^{4}$ J. B. G. M. Bloemen, ${ }^{5,6}$ R. Buccheri, ${ }^{7}$ P. A. Caraveo, ${ }^{4}$ M. Gottwald,${ }^{8}$ W. Hermsen, ${ }^{5}$ G. Kanbach,${ }^{8}$ H. A. Mayer-Hasselwander,${ }^{8}$ T. Montmerle, ${ }^{1}$ J. A. Paul, ${ }^{1}$ B. Sacco, ${ }^{2}$ A. W. Strong,${ }^{4}$ and R. D. Wills ${ }^{3}$ Caravane Collaboration for the $\operatorname{COS} B$ Satellite

AND

T. M. Dame, R. S. Cohen, And P. Thaddeus

Goddard Institute for Space Studies; and Columbia University Received 1983 February 2; accepted 1983 April 6

\begin{abstract}
The fully sampled Columbia $1^{\circ} \mathrm{CO}$ survey and the Berkeley $\mathrm{H}$ I survey are compared with the $\operatorname{COS} B$ gamma-ray survey. As a first step, the study is limited to the high-energy gamma-rays $(E>300 \mathrm{MeV})$. It is found that a simple model, in which uniformly distributed cosmic rays interact with the interstellar gas, as traced by $\mathrm{H}$ I and $\mathrm{CO}$, can account for almost all the observed gamma-rays. Furthermore, if the contribution from point sources to the gamma-ray flux is significant, these sources must have a galactic distribution similar to that of $\mathrm{CO}$. The analysis permits calibration of the ratio between $\mathrm{H}_{2}$ column density and integrated $\mathrm{CO}$ line intensity: $N\left(\mathrm{H}_{2}\right) / W_{\mathrm{CO}}=(1-3) 10^{20}$ molecules $\mathrm{cm}^{-2} \mathrm{~K}^{-1} \mathrm{~km}^{-1} \mathrm{~s}$.
\end{abstract}

Subject headings: cosmic rays: general - gamma rays: general — interstellar: molecules radio sources: $21 \mathrm{~cm}$ radiation

\section{INTRODUCTION}

Although many surveys at radio and millimeter wavelengths have been undertaken in recent years, the relative contribution of $\mathrm{H}_{2}$ to the total gas content of the Galaxy is still an open question (see, e.g., Lequeux 1981). It has been thought for some time that the galactic gamma-ray emission results mainly from the interactions of ambient cosmic rays (CRs) with the interstellar gas and is therefore potentially a valuable gas tracer. However, the possibility of a galactic CR gradient and the discovery of many gamma-ray sources, such as the Crab and Vela pulsars, have complicated this simple picture. Nevertheless because the problems encountered in interpreting the gamma-ray and millimeter-wave observations are independent, a detailed comparison of $\mathrm{CO}$ and gammaray surveys should shed some light on the galactic distribution of interstellar gas and CRs and on the gamma-ray point-source contribution.

\footnotetext{
${ }^{1}$ Service d'Astrophysique, Centre d'Etudes Nucléaires de Saclay, France.

${ }^{2}$ This work was done while the author was at the Goddard Institute for Space Studies.

${ }^{3}$ Space Science Department of the European Space Agency, ESTEC, Noordwijk, The Netherlands.

${ }^{4}$ Istituto di Fisica Cosmica del CNR, Milano, Italy.

${ }^{5}$ Cosmic-Ray Working Group, Huygens Laboratorium, Leiden, The Netherlands.

${ }^{6}$ Also Sterrewacht, Huygens Laboratorium, Leiden, The Netherlands.

${ }^{7}$ Istituto di Fisica Cosmica e Informatica del CNR, Palermo, Italy.

${ }^{8}$ Max-Planck-Institut für Physik und Astrophysik, Institut für Extraterrestrische Physik, Garching bei München, Germany.
}

The COS $B$ satellite has provided a complete picture of the Milky Way at latitudes less than $25^{\circ}$ in high-energy gamma-rays (Mayer-Hasselwander et al. 1982): The $1.2 \mathrm{~m} \mathrm{CO}$ survey telescope at Columbia University has been used to perform a fully-sampled $\mathrm{CO}$ survey over a large latitude range in the first galactic quadrant (Dame and Thaddeus 1983). The large extent of this survey allows for the first time a detailed comparison of the gamma-ray intensity with the gas distribution $-\mathrm{H}_{2}$ as traced by $\mathrm{CO}$ and $\mathrm{H}$ I as traced by the $21 \mathrm{~cm}$ line. This article presents the results of such a comparison, restricted to the high-energy gamma-rays $(E>300$ $\mathrm{MeV}$ ), from the first galactic quadrant.

\section{DATA AND ANALYSIS}

The CO survey of Dame and Thaddeus (1983) has an angular resolution of $1^{\circ}$, adequate for comparison with available gamma-ray data. This wide beam allows for fast and complete sampling over extended regions of the sky. The observations have been made from $l=11.5$ to $97^{\circ} .5$ and from $b=-5.5$ to 10.5 . However, the latitude range $6.5-10.5$ is not completely covered for all longitudes (only about $53 \%$ ). The spectra have been integrated over all velocities, yielding a quantity hereafter referred to as $W_{\mathrm{CO}}$. The $\mathrm{H}$ I data have been taken mainly from the $21 \mathrm{~cm}$ line survey of Weaver and Williams (1973), supplemented by the medium latitude survey of Heiles and Habing (1974) (for $|b|>10^{\circ}$ ). While a spin temperature of $135 \mathrm{~K}$ has been adopted in deriving the $\mathrm{H}$ I column densities from the Weaver and Williams survey $\left(|b|<10^{\circ}\right)$, the $21 \mathrm{~cm}$ line has been assumed to 
be optically thin for $\left(|b|>10^{\circ}\right)$. The gamma-ray data used are described by Mayer-Hasselwander et al. (1982) and refer to $36 \operatorname{COS~} B$ observation periods of approximately 1 month duration each. These data cover the entire galactic plane with a statistical accuracy so far unknown in this energy domain. In order to compare the $\operatorname{COS} B$ gamma-ray data with the gas tracer surveys, it is necessary to reduce the latter to the gamma-ray angular resolution by means of a convolution with the point spread function (PSF) of the $\operatorname{COS} B$ telescope. By restricting the gamma-ray data to $E>300 \mathrm{MeV}$, where the FWHM of the PSF is $\sim 2.3$, we minimize the contribution to the gamma-ray intensity observed inside the $\mathrm{CO}$ survey area from zones outside those mapped in $\mathrm{CO}$.

Since the derivation of an $\mathrm{H}_{2}$ column density from $\mathrm{CO}$ measurements involves many uncertainties (see, e.g., Lequeux 1981), we simply attempted to reproduce the observed gamma-ray emission by finding the best values of the parameters $A, B$, and $C$ in the expression

$$
I_{P}=N_{P} / \Omega t=A N(\mathrm{H} \mathrm{I})+B W_{\mathrm{CO}}+C,
$$

where $I_{P}$ and $N_{P}$ are the predicted gamma-ray rate and counts, $\Omega$ is the geometrical factor, and $t$, the integration time. $N\left(\mathrm{H} \mathrm{I}_{\mathrm{I}}\right)$ and $W_{\mathrm{CO}}$ refer to the quantities obtained after convolving the $\mathrm{H}$ I and $\mathrm{CO}$ data with the PSF for $E>300 \mathrm{MeV}$, on the assumption that the $\mathrm{CO}$ emission in the regions not observed (shaded areas in Fig. 3) is equal to that (generally rather weak) measured in adjacent directions. The values of $A, B$, and $C$ were adjusted to produce a predicted gamma-ray map which maximizes the probability of obtaining the observed map. In other words, the likelihood

$$
L=\prod_{i, j}\left[N_{P}^{\left(\mathrm{N}_{0}\right)} \exp \left(-N_{P}\right) /\left(N_{0} !\right)\right],
$$

[where $N_{0}$ is the observed gamma-ray count in bin $(i, j)$ the product is extended over the available sky] has been computed, and the $(A, B, C)$ set which maximizes $L$ has been chosen as the best estimate. The maximum likelihood estimates of parameters $A, B$, and $C$ are given in Table 1.

Our parameters $A$ and $C$ have the same physical meaning as the parameters $A_{2}$ and $B_{2}$ derived in Lebrun et al. (1982). These later parameters, holding for the solar neighborhood, were derived using the interstellar absorption to trace the gas column density and following a statistical procedure similar to that used here. In view of the systematic uncertainties which affect independently both analysis, the differences observed in Table 1 do not appear significant.
The more recent estimate of the gamma-ray emissivity in the solar neighborhood by Strong et al. (1982) is in good agreement with the values given in Table 1 .

When performing a multilinear regression, one has to ensure that the variables are linearly independent. The advantage of the maximum likelihood method is that the uncertainties derived for each parameter take into account the degree of dependence of the variables. In the present context, it appears that $W_{\mathrm{CO}}$ and $N(\mathrm{H} \mathrm{I})$ are practically linearly independent. This is illustrated in Figure 1, where the gamma-ray emission expected from $\mathrm{H}$ I alone (dotted line) differs significantly from that expected from $\mathrm{H}$ I and $\mathrm{CO}$ together (solid line).

In Figures 1, 2, and 3, the predicted gamma-ray emission is compared with that actually observed. Figure $1 a$ shows clearly that it is possible to reproduce simultaneously the gamma-ray emission in directions near the galactic center and well away from it. Figure $1 b$ demonstrates that this agreement in the plane is maintained at medium latitudes in all directions. The agreement between the latitude distributions of the observed and predicted gamma-ray emission for various longitude ranges is apparent in Figure 2. It should be noted that the width of these distributions is much larger than that expected from emission confined to $b=0^{\circ}$ (thin line) and that the asymmetries around the plane are also reproduced. The two-dimensional agreement can be seen in Figure 3 which displays contour maps of the observed and predicted gamma-ray emission. A quantitative study of the differences between the observed and the predicted maps shows that the most significant deviation occurs in the Cygnus region $\left(l: 75^{\circ}-85^{\circ}\right)$. Although the angular distribution of the gamma-ray emission in this region is compatible with the presence of compact sources (Swanenburg et al. 1981), this excess may also result from an underestimate of the amount of molecular gas owing to the strong velocity crowding in this region.

\section{DISCUSSION}

The good overall agreement between the gamma-ray emission and the gas tracers might seem surprising in view of the transparency of the interstellar medium to gamma rays and the high optical depth of the CO $1 \rightarrow 0$ line in many molecular clouds. However, because of differential galactic rotation and cloud clumpiness, shadowing of one cloud by another is unlikely, and $W_{\text {CO }}$ is therefore probably a good tracer of the number of $\mathrm{CO}$ clouds along the line of sight. Furthermore, the fact that the $\mathrm{CO}$ lines are roughly proportional to the less saturated ${ }^{13} \mathrm{CO}$ lines in molecular clouds (see e.g., Solomon and Sanders 1980) that are known from star

TABLE 1

\begin{tabular}{|c|c|c|}
\hline Parameter & This Work & Lebrun et al. (1982) \\
\hline $\begin{array}{l}A\left[10^{-25} \text { photons }(\mathrm{H} \text { atom })^{-1} \mathrm{~cm}^{2} \mathrm{~s}^{-1} \mathrm{sr}^{-1}\right] \ldots \ldots \\
B\left(10^{-4} \text { photons } \mathrm{sr}^{-1} \mathrm{~K}^{-1} \mathrm{~km}^{-1}\right) \ldots \ldots \ldots \ldots \ldots \ldots \ldots \\
C\left(10^{-4} \text { photons } \mathrm{s}^{-1} \mathrm{sr}^{-1}\right) \ldots \ldots \ldots \ldots \ldots \ldots \ldots \ldots \ldots \ldots\end{array}$ & $\begin{array}{r}2.81+0.48 \\
1.75_{-0.25}^{+0.39} \\
10.9 \pm 1.3\end{array}$ & $\begin{array}{c}2.14 \pm 0.27 \\
\cdots \\
8.17 \pm 0.59\end{array}$ \\
\hline
\end{tabular}

Best Parameter Values 

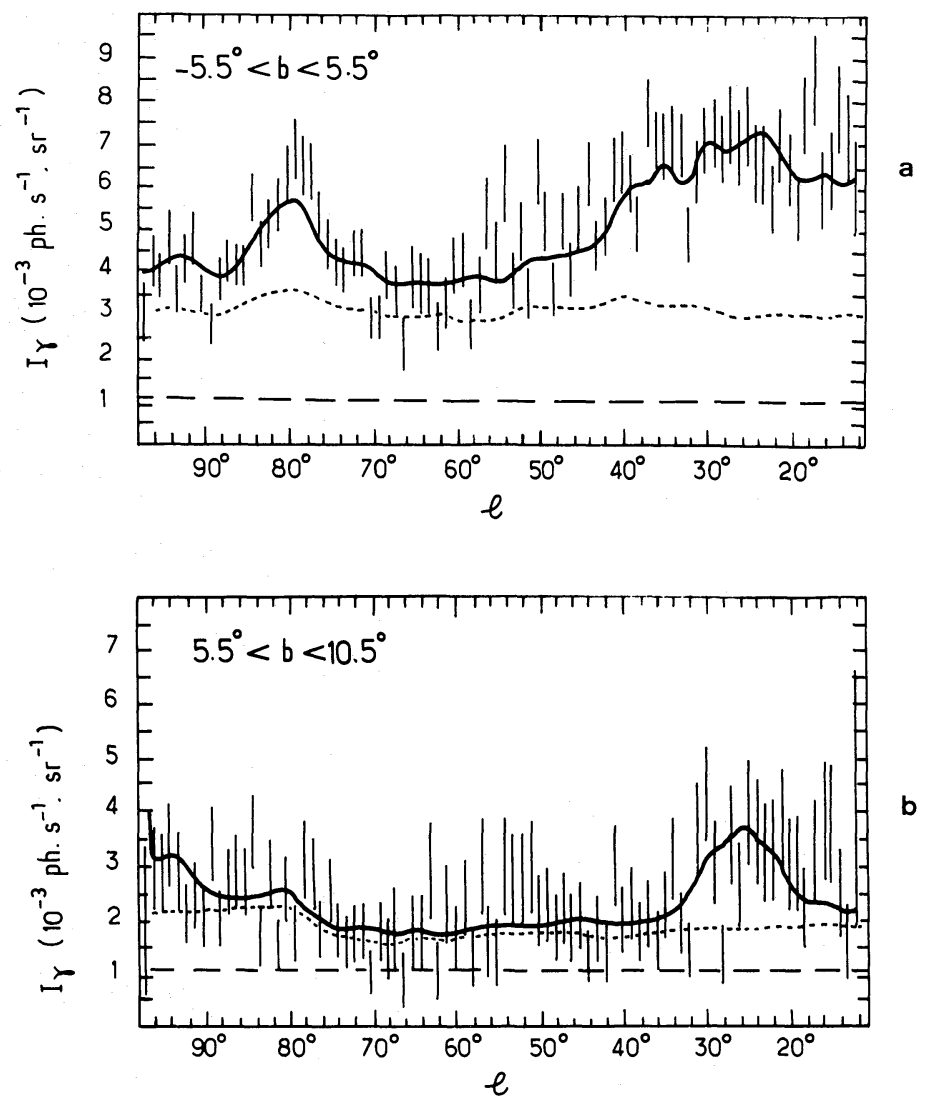

FIG. 1.-Longitude profiles of the observed gamma-ray emission for $E>300 \mathrm{MeV}$ (error bars) compared with that expected from CO plus $\mathrm{H}$ I (thick line) and from $\mathrm{H}$ I alone (dotted line). The included background level $C$ is indicated by the interrupted line. Note that the $\mathrm{CO}$ survey is not complete for $l: 12^{\circ}-20^{\circ}$ and $l: 55^{\circ}-97^{\circ}$ at medium latitudes (see Fig. 3).

count data to trace the $\mathrm{H}_{2}$ mass (Dickman 1978) suggests that $W_{\mathrm{CO}}$ is roughly proportional to the column density of $\mathrm{H}_{2}$, despite its high optical depth.

The gamma-ray intensity may be considered as the sum of contributions from (i) CR (both electrons and nuclei) interactions with atomic gas, primarily $\mathrm{H} \mathrm{I}: I_{\mathrm{HI}} ;(i i) \mathrm{CR}$ interactions with molecular gas, primarily $\mathrm{H}_{2}: I_{\mathrm{H}_{2}}$; (iii) localized sources: $I_{\mathrm{LS}}$; (iv) extragalactic emission: $I_{E}$; and $(v) \operatorname{COS} B$ instrumental background: $I_{\mathrm{BG}}$. Gamma-rays can also be produced by the inverse Compton scattering of high-energy electrons on the ambient photon field. However, the energy spectrum of these electrons is very steep in the relevant range: various calculations show that this mechanism may contribute to low- or medium-energy gamma-rays (Kniffen and Fichtel 1981) but not significantly above $300 \mathrm{MeV}$.

It is reasonable to assume that $I_{\mathrm{H}}$ is distributed like $N(\mathrm{H} \mathrm{I})$ and that $I_{\mathrm{H}_{2}}$ is distributed like $W_{\mathrm{CO}}$. The extragalactic emission is small and dominated by the isotropic $\operatorname{COS} B$ instrumental background. Assuming that the localized source contribution is negligible, the three terms of equation (1), $A N(\mathrm{H} \mathrm{I}), B W_{\mathrm{CO}}$, and $C$ can be identified respectively with $I_{\mathrm{H}}, I_{\mathrm{H}_{2}}$, and $I_{\mathrm{BG}}$. If we further assume that $q$, the gamma-ray emissivity per $\mathrm{H}$ atom is uniform within the Galaxy ${ }^{9}$ and over the various phases of the interstellar medium, the gamma-ray rates expected from atomic and molecular hydrogen are, respectively: $I_{\mathrm{HI}}=(q S / 4 \pi) N(\mathrm{H} \mathrm{I})$ and $I_{\mathrm{H}_{2}}=(q S / 4 \pi) 2 N\left(\mathrm{H}_{2}\right)$ (where $S$ is the effective sensitive area of the $\operatorname{COS} B$ experiment and $N\left(\mathrm{H}_{2}\right)$ is the column density of hydrogen molecules). Consequently $A=q S / 4 \pi$ and $B=(q S / 4 \pi) 2 N\left(\mathrm{H}_{2}\right) / W_{\mathrm{CO}}$, so that $N\left(\mathrm{H}_{2}\right) / W_{\mathrm{CO}}=B / 2 A$. Thus in the simple case where the $C R$ density is uniform throughout the galactic disk and the localized source contribution is negligible, we have $N\left(\mathrm{H}_{2}\right) / W_{\mathrm{CO}}=X=3 \times 10^{20}$ molecules $\mathrm{cm}^{-2}$ $\mathrm{K}^{-1} \mathrm{~km}^{-1} \mathrm{~s}$. In other words, gamma-rays are used as a standard, which is first calibrated itself on $\mathrm{H} \mathrm{I}$ and then used to calibrate the $\mathrm{H}_{2}$ estimates from $\mathrm{CO}$ measurements. At this point, it should be stressed that this derivation relies on our ability to determine independently $A$ and $B$. From Figure $1 a$, it is clear that $B$ can be determined alone since $A N(\mathrm{H} \mathrm{I})$ is almost constant. Then $A$ can be determined by the latitude variation of $I_{0}-B W_{\mathrm{CO}}$ (where $I_{0}$ is the observed gamma-ray rate).

${ }^{9}$ The assumption of the uniformity of the gamma-ray emissivity on a galactic scale is substantiated when noting the similarities between the results obtained in the solar vicinity (Lebrun et al. 1982; Strong et al. 1982) and those presented here. 


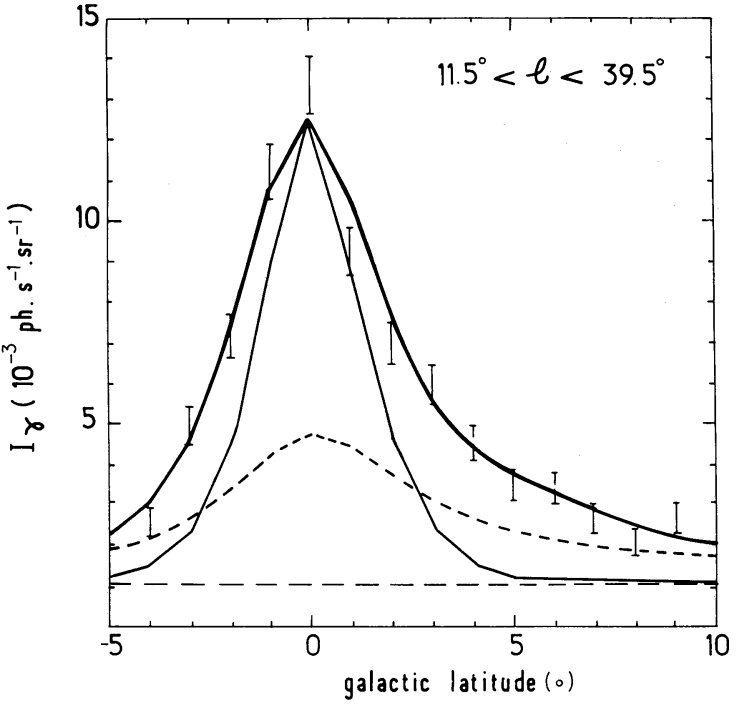

FIG. $2 a$

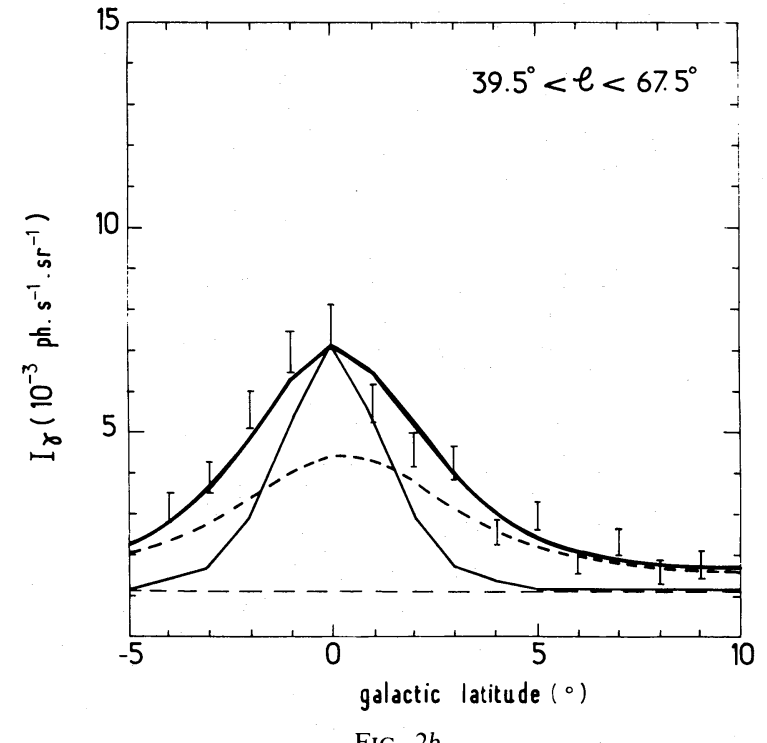

FIG. $2 b$

FIG. 2.-Latitude profiles of the observed gamma-ray emission for $E>300 \mathrm{MeV}$ (error bars) compared with that expected from CO plus $\mathrm{H} \mathrm{I}$ (thick line) and from $\mathrm{H}$ I alone (dotted line). The thin line shows the profile expected from emission confined to $b=0^{\circ}$, scaled arbitrarily to the gamma-ray emission expected from $\mathrm{CO}$ plus $\mathrm{H}$ i at $b=0^{\circ}$.

The high-latitude data constrain primarily $C$, while the low-latitude data fix $A$.

We consider now the effect of a nonuniform CR density and localized sources. A strong CR-gradient is unlikely because it would produce a longitudinal variation of the gamma-ray intensity larger than that actually observed unless one invokes a variation of $X$ inverse to that of the cosmic rays, such that the product $q X$ remains constant. Furthermore, a strong gradient would have produced a significant difference between the emissivity parameters derived here and by Lebrun et al. (1982) and Strong et al. (1982). A small CR gradient would not significantly affect the derived $X$ value, because it would affect the $A$ and $B$ coefficients by comparable amounts. The galactic gamma-ray source distribution is remarkably similar to the $\mathrm{CO}$ distribution (Swanenburg et al. 1981). It is then likely that $I_{\mathrm{LS}}$ contributes to $B W_{\mathrm{CO}}$ which is similarly peaked along the galactic plane; hence $I_{\mathrm{H}_{2}}=B W_{\mathrm{CO}}-I_{\mathrm{LS}}$. Any contribution from the gamma-ray sources to $A N(\mathrm{H} \mathrm{I})$ is ruled out by the large difference between the latitude distributions of the gamma-ray sources and $\mathrm{H} \mathrm{I}$.

Consequently as any source contribution effectively increases the value of $X$ as derived above, it should be considered an upper limit. This may be expressed as $X=(B / 2 A)(1-F)$, with $F=I_{\mathrm{LS}} /\left(B W_{\mathrm{CO}}\right)$ and $0<F<1$. In the absence of other constraints, $B W_{\mathrm{CO}}$ could be entirely accounted for by the localized sources. However, since the typical distance of $\operatorname{COS} B$ gamma-ray sources is larger than $2 \mathrm{kpc}$ and because sources are more easily detected toward the galactic anticenter, all localized sources within a few $\mathrm{kpc}$ in this direction should be in the 2CG catalog (Swanenburg et al. 1981). If the localized source contribution mimics that of $\mathrm{H}_{2}$, it falls off outside a few kpc in the anticenter direction. It is then extremely

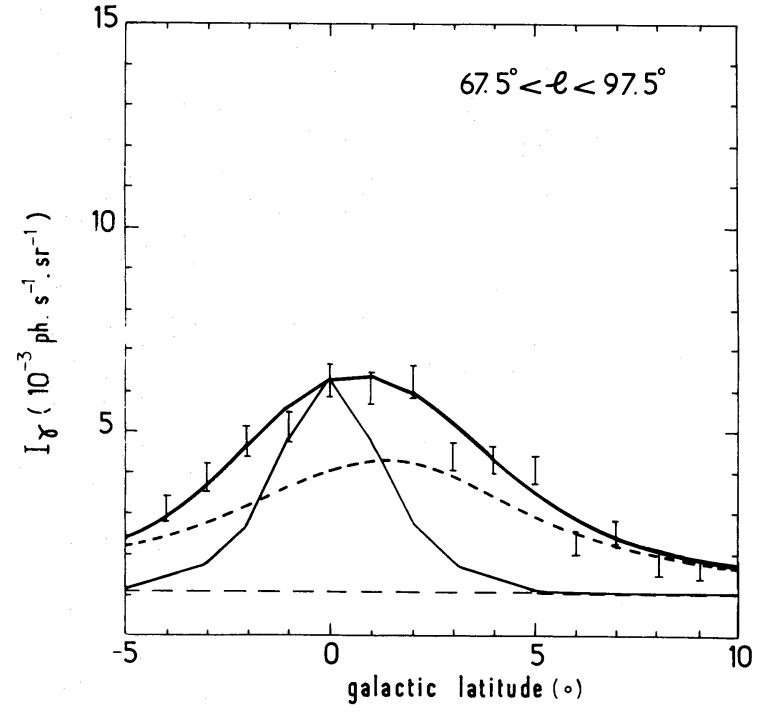

FIG. $2 c$

unlikely that any localized source of luminosity $\sim 10^{35}$ ergs $\mathrm{s}^{-1}$, typical of the $2 \mathrm{CG}$ catalog, have been missed in this direction. Since some of these $2 \mathrm{CG}$ sources may be associated with dense molecular clouds, the contribution of the $2 \mathrm{CG}$ sources to the gamma-ray emission in the anticenter should be regarded as an upper limit to the pointlike source contribution in this direction. However, since the Vela pulsar alone accounts for $60 \%$ of the emission, this upper limit is rather uncertain.

A lower limit to $X$ may then be obtained by subtracting the maximum localized source contribution from $B W_{\mathrm{CO}}$. If the emission from localized sources for $90^{\circ}<l<270^{\circ}$ is set to $4 \times 10^{-4}$ photons $\mathrm{s}^{-1}$ (i.e., the $2 \mathrm{CG}$ source 


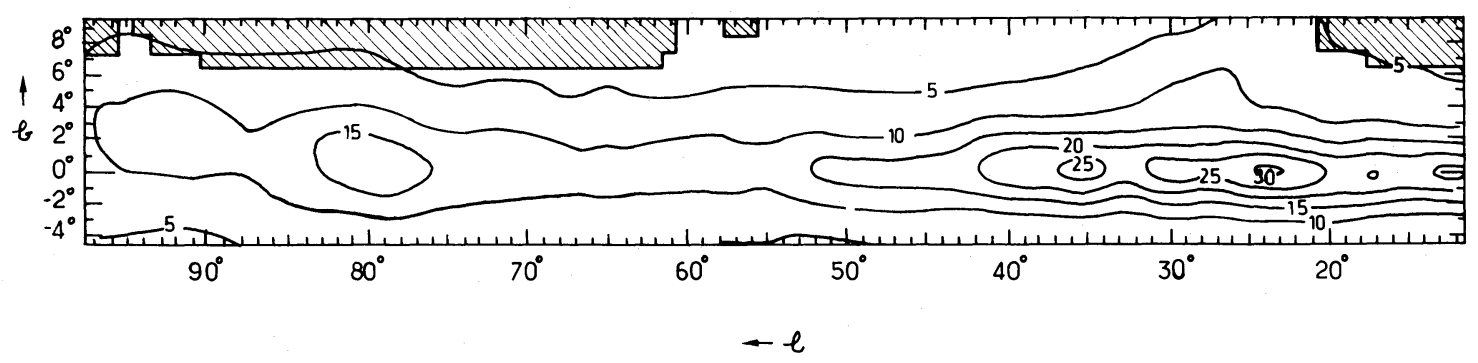

FWHM of the

COS-B PSF $(E>300 \mathrm{MeV})$

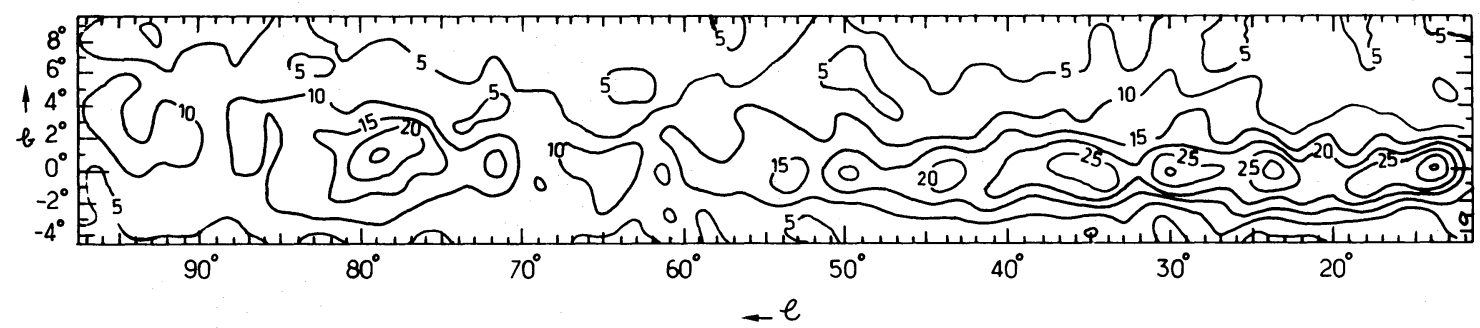

FIG. 3.-Contour maps of the predicted (upper part) and observed (lower part) gamma-ray emission for $E>300 \mathrm{MeV}$. The contour interval is: $4.7 \times 10^{-4}$ photons s${ }^{-1} \mathrm{sr}^{-1}$. The shaded areas represent regions unobserved in $\mathrm{CO}$. Because of the different statistical accuracies of the $\mathrm{CO}$ and gamma-ray observations, a very detailed comparison of the two maps is not warranted. For the limitations of the gamma-ray map see Mayer-Hasselwander et al. (1982).

emission above $300 \mathrm{MeV}$ in this longitude range), their relative contribution to the gamma-ray emission is $70 \%$ of $B W_{\mathrm{CO}}(F=0.7)$ and $X$ is reduced to $1 \times 10^{20}$ molecules $\mathrm{cm}^{-2} \mathrm{~K}^{-1} \mathrm{~km}^{-1} \mathrm{~s}$. Although this result is somewhat imprecise, it illustrates the range of uncertainty caused by localized sources.

A comparison with independent determinations of $X$ is appropriate, using radio and interstellar extinction measurements in dark clouds, Dickman (1978) derived the ratio $N\left(\mathrm{H}_{2}\right) / N\left({ }^{13} \mathrm{CO}\right)=5 \times 10^{5}$. However if the $W_{\text {CO }}$ values measured by Dickman are used instead of the derived $N\left({ }^{13} \mathrm{CO}\right)$ values, an $X$ value of $1 \times 10^{20}$ molecules $\mathrm{cm}^{-2} \mathrm{~K}^{-1} \mathrm{~km}^{-1} \mathrm{~s}$ is obtained. This value can be considered a lower limit since a higher value is indicated by all measurements with $A_{v}>2 \mathrm{mag}$, a range of absorption which seems more appropriate for a galactic survey of molecular clouds. In particular the observations of much more obscured regions in the $\rho$ Ophiuchi cloud (Frerking, Langer, and Wilson 1982) imply $X=1.8 \times 10^{20}$ molecules $\mathrm{cm}^{-2} \mathrm{~K}^{-1} \mathrm{~km}^{-1} \mathrm{~s}$.

In summary, the study of the correlation between the galactic gamma-ray and $\mathrm{CO}$ emission has provided a calibration of the ratio

$N\left(\mathrm{H}_{2}\right) / W_{\mathrm{CO}}=1-3 \times 10^{20}$ molecules $\mathrm{cm}^{-2} \mathrm{~K}^{-1} \mathrm{~km}^{-1} \mathrm{~s}$,

which agrees with the local estimates based on interstellar extinction measurements. Since about half of the CO emission in the Dame and Thaddeus survey occurs locally, the above mentioned calibration should be considered a galactic average weighted almost equally by the local emission and that of the molecular ring.

The molecular hydrogen content of the inner Galaxy has been derived by Solomon and Sanders (1980) on the basis of an undersampled CO survey. They claimed that between 4 and $8 \mathrm{kpc}$ from the galactic center, the molecular component is dominant. This has been recently challenged by Cohen et al. (1980) who proposed an approximate equipartition of the mass in atoms and molecules. The approach of Solomon and Sanders assumes a proportionality between $N\left(\mathrm{H}_{2}\right)$ and $N\left({ }^{13} \mathrm{CO}\right)$ and between $N\left({ }^{13} \mathrm{CO}\right)$ and $W_{13} \mathrm{Co}$. Observing that $W_{13} \mathrm{CO}$ is roughly proportional to $W_{\mathrm{CO}}$, they concluded that $W_{\mathrm{CO}}$ is also a tracer of $N\left(\mathrm{H}_{2}\right)$. Solomon and Sanders's statement on the preponderance of molecular hydrogen ultimately relies on the ratio $N\left(\mathrm{H}_{2}\right) / W_{\mathrm{CO}}=6 \times 10^{20}$ molecules $\mathrm{cm}^{-2} \mathrm{~K}^{-1} \mathrm{~km}^{-1} \mathrm{~s}$, which is outside the acceptable range derived in this letter. This implies that the molecular content of the inner Galaxy given by Solomon and Sanders is overestimated by at least a factor of 2 and even more if pointlike sources make a significant contribution to the overall gamma-ray emission. This result agrees with the findings of Federman and Evans (1981) from a reanalysis of observations towards the galactic center at different wavelengths. Finally, we note that the more recent $X$ value proposed by Sanders (1982), $X=3.6 \times 10^{20}$ molecules $\mathrm{cm}^{-2} \mathrm{~K}^{-1} \mathrm{~km}^{-1} \mathrm{~s}$, is almost consistent with our upper limit, although he suggests that this value is probably an underestimate. 


\section{REFERENCES}

Cohen, R. S., Cong, H., Dame, T. M., and Thaddeus, P. 1980, Ap. J. (Letters), 239, L53.

Dame, T. M., and Thaddeus, P. 1983, in preparation.

Dickman, R. L. 1978, Ap. J. Suppl., 37, 407.

Federman, S. R., and Evans, N. J. 1981, Ap. J., 248, 113.

Frerking, M. A., Langer, W. D., and Wilson, R. W. 1982, Ap. J., 262, 590.

Heiles, C., and Habing, H. J. 1974, Astr. Ap. Suppl., 14, 1.

Kniffen, D. A., and Fichtel, C. E. 1981, Ap. J., 250, 389.

Lebrun, F., et al. 1982, Astr. Ap., 107, 390.
Lequeux, J. 1981, Comments Ap., 9, 117.

Mayer-Hasselwander, H. A., et al. 1982, Astr. Ap., 105, 164.

Sanders, D. B. 1982, Ph.D. thesis, State University of New York.

Solomon, P. M., and Sanders, D. B. 1980, in Giant Molecular Clouds in the Galaxy, ed. P. M. Solomon and E. G. Edmunds (Elmsford, N.Y.: Pergamon Press), p. 41.

Strong, A. W., et al. 1982, Astr. Ap., 115, 404.

Swanenburg, B. N., et al. 1981, Ap. J. (Letters), 243, L69.

Weaver, H. J., and Williams, R. W. 1973, Astr. Ap. Suppl., 8, 1.

K. BennetT and R. D. Wills: Space Science Department, European Space Agency, Estec, Noordwijk, The Netherlands

G. F. Bignami, P. A. Caraveo, and A. W. Strong: Istituto di Fisica Cosmica del CNR, 15, Via Bassini, 20133 Milan, Italy

J. B. G. M. Bloemen and W. Hermsen: Cosmic Ray Working Group, Huygens Laboratorium, Wassenaarseweg 78, Leiden, The Netherlands

R. BuCCHERI and B. SACCO: Istituto di Fisica Cosmica e Informatica del CNR, Via Archirafi 36, Palermo, Italy

R. S. Cohen, T. M. Dame, and P. Thaddeus: Goddard Institute for Space Studies, 2880 Broadway, New York, NY 10025

M. Gottwald, G. Kanbach, and H. A. Mayer-Hasselwander: Max-Planck-Institut für Extraterrestrische Physik, 8046 Garching bei München, German Federal Republic

F. Lebrun, T. Montmerle, and J. A. Paul: Service d'Astrophysique, Centre d'Etudes Nucléaires de Scalay, 91191 Gif-sur-Yvette, Cedex, France 